\title{
Campylobacter résistant à la ciprofloxacine dans le poulet à griller au Canada
}

\author{
Agunos A, ${ }^{1 *}$ Léger DF, ${ }^{1}$ Avery BP, ${ }^{1}$ Parmley EJ, ${ }^{1}$ Deckert AE, ${ }^{1}$ Carson CA, ${ }^{1}$ Reid-Smith RJ, ${ }^{1}$ \\ Irwin RJ ${ }^{1}$
}

${ }^{1}$ Laboratoire de lutte contre les zoonoses d'origine alimentaire, Agence de la santé publique du Canada, Guelph (Ontario)

* Correspondance à : agnes.agunos@phac-aspc.gc.ca

\section{Résumé}

La présente étude de cas décrit les tendances de la résistance à la ciprofloxacine des isolats de Campylobacter provenant de viande de poulet vendue au détail au Canada. Campylobacter vient au troisième rang des maladies entériques d'origine alimentaire les plus courantes au Canada. Elle entraîne habituellement une maladie spontanément résolutive, mais l'usage d'antimicrobiens peut être indiqué dans certains cas. La ciprofloxacine (une fluoroquinolone) est un agent antimicrobien utilisé pour traiter un certain nombre d'infections chez les humains; d'autres fluoroquinolones sont utilisées à des fins préventives ou de traitement chez les animaux d'élevage, y compris les poulets à griller. Depuis 2003, le Programme intégré canadien de surveillance de la résistance aux antimicrobiens (PICRA) effectue des tests sur des échantillons de viande de poulet vendue au détail à l'échelle du Canada afin de détecter la présence de Campylobacter et de souches résistantes. À la fin de 2010, le PICRA signalait que les échantillons de viande de poulet vendue au détail au Canada qui étaient contaminés par Campylobacter variaient de $36 \%$ dans la région des Maritimes à $42 \%$ en Colombie-Britannique. De plus, les niveaux de Campylobacter résistant à la ciprofloxacine variaient dans l'ensemble du pays, avec des pourcentages plus élevés en Colombie-Britannique (17\% en 2010) et en Saskatchewan (11\%), en comparaison avec des pourcentages plus faibles en Ontario (5\%), au Québec (2\%), et dans la région des Maritimes (4\%). En 2011 et en 2012, la résistance diminuait en Colombie-Britannique et en Saskatchewan, mais commençait à augmenter au Québec et en Ontario. Récemment, l'industrie canadienne de la volaille a élaboré une politique visant à éliminer l'utilisation préventive des céphalosporines de troisième génération et des fluoroquinolones chez les poules reproductrices de type à griller (poules qui pondent les œufs qui deviendront les poulets à griller). Comme suite à cette intervention de l'industrie, le PICRA continuera à surveiller les tendances quant à l'utilisation des antimicrobiens et la résistance à ceux-ci. En adoptant de bonnes pratiques d'hygiène et de préparation de la nourriture, les Canadiens peuvent réduire leur risque de contracter une infection à Campylobacter (résistante ou vulnérable aux antimicrobiens) provenant de la viande de poulet vendue au détail.

\section{Introduction}

Les antimicrobiens sont couramment utilisés pour le traitement d'une multitude de maladies infectieuses et de problèmes de santé chez les humains et les animaux. La présente étude de cas décrit les tendances de la résistance à la ciprofloxacine parmi les isolats de Campylobacter provenant de la viande de poulet vendue au détail au Canada, examine les répercussions éventuelles de cette résistance sur la santé humaine et dresse la liste des mesures prises pour y remédier. Elle décrit la façon dont le système de surveillance " de la ferme à la table » du Canada, le Programme intégré canadien de surveillance de la résistance aux antimicrobiens (PICRA), a détecté ce profil de résistance et en a fait le suivi alors qu'il émergeait d'un bout à l'autre du pays. Nous avons également déterminé les répercussions de cette information sur l'utilisation des médicaments antimicrobiens au sein de l'industrie canadienne de la volaille et les mesures supplémentaires qui sont prises afin de mieux comprendre et de réduire cette menace à la santé humaine et animale. 


\section{Campylobacter}

Des estimations récentes indiquent qu'environ $90 \%$ des 1,6 million de cas estimés de maladies d'origine alimentaire contractées au Canada sont causées par les quatre agents pathogènes suivants : Norovirus, Clostridium perfringens, Campylobacter et Salmonella non typhique $(1,2)$. La plupart des infections à Campylobacter sont généralement spontanément résolutives, causant de la diarrhée, des crampes abdominales, de la fièvre, des maux de tête et de la myalgie.

On croit que les infections à Campylobacter sont acquises principalement par la consommation de produits alimentaires contaminés, une plus faible proportion de cas étant causés par un contact direct avec des animaux de ferme ou de compagnie, ou de l'eau contaminée de zones récréatives. Des études cas-témoins ont révélé un lien important entre la campylobactériose chez les humains et la consommation de volaille crue ou insuffisamment cuite (3).

En général, un traitement à l'aide d'antimicrobiens n'est pas nécessaire pour les cas simples, mais il peut l'être pour le traitement des patients vulnérables ou pour les infections graves, systémiques ou prolongées. Lorsqu'un traitement est nécessaire, les médicaments à base de macrolides (érythromycine ou azithromycine) sont recommandés, la ciprofloxacine étant utilisée comme solution de rechange (4). Toutefois, une étude sur l'utilisation des antimicrobiens et la résistance à ceux-ci dans deux unités sanitaires en Ontario a révélé que 45 des 138 cas humains atteints de campylobactériose avaient utilisé de la ciprofloxacine (5).

\section{Ciprofloxacine}

La ciprofloxacine est un antimicrobien à base de fluoroquinolones couramment utilisée chez les humains pour le traitement des infections respiratoires, urinaires, cutanées et osseuses/articulaires, de même que pour le traitement de certains cas de gastroentérite chez les adultes (6). Les fluoroquinolones sont considérées comme étant « extrêmement » ou « très » importantes en médecine humaine (catégorie I) par l'Organisation mondiale de la Santé (7) et la Direction des médicaments vétérinaires de Santé Canada [8].

Les fluoroquinolones sont utilisées uniquement lors d'éclosions de maladies bactériennes rares ou sporadiques chez les poulets, ou pour le traitement d'infections graves où l'utilisation d'antimicrobiens fréquemment utilisés s'est avérée inefficace (c'est-à-dire lorsque l'on recense un nombre croissant d'oiseaux malades ou morts dans l'élevage malgré l'utilisation d'antimicrobiens) (9). Au Canada, seule l'enrofloxacine est homologuée pour son utilisation chez les bovins, les porcs, les chiens et les chats, et la danofloxacine n'est autorisée que pour les bovins. Ces antimicrobiens de la famille des fluoroquinolones ne sont pas approuvés pour la volaille. Selon la Direction des médicaments vétérinaires de Santé Canada, cette classe d'antimicrobiens ne doit pas être utilisée comme médicament de masse (c.-à-d. pour traiter un troupeau entier de volaille ou d'animaux) en dérogation des directives de l'étiquette (c.-à-d. d'une manière qui n'est pas conforme à l'étiquette ou à la notice d'accompagnement du produit) (10).

\section{Surveillance de la résistance aux antimicrobiens}

L'Agence de la santé publique du Canada (I'Agence) est responsable de la coordination du PICRA, un système de surveillance de l'utilisation des antimicrobiens chez les humains et les animaux et de la résistance aux antimicrobiens (RAM) de certaines bactéries détectées chez les humains, les animaux et les aliments (11). La composante agroalimentaire de ce programme comporte les éléments suivants : la surveillance, à l'échelle des troupeaux de bovins ou de volaille, des données sur l'utilisation des antimicrobiens et sur la RAM dans des fermes sentinelles; la surveillance en abattoir, en fournissant des données nationales représentatives de la RAM à partir de bactéries d'animaux prélevées à l'entrée de ces derniers dans l'approvisionnement alimentaire; la surveillance de la viande vendue au détail, en fournissant des données sur la RAM à partir de bactéries observées dans la viande crue achetée dans les magasins d'alimentation, révélant l'exposition du consommateur aux bactéries résistantes aux antimicrobiens dans les aliments. L'Institut canadien de la santé animale fournit également au PICRA les 
quantités d'antimicrobiens distribués pour la vente et destinés aux animaux au Canada. Les bactéries d'intérêt sont du genre Salmonella, Escherichia coli génériques et Campylobacter.

\section{Détection de Campylobacter résistant à la ciprofloxacine dans la viande de poulet vendue au détail}

Depuis, 2003, le PICRA effectue des tests sur des échantillons de viande de poulet vendue au détail partout au Canada afin de détecter la présence de la bactérie Campylobacter. Ces études ont commencé en Ontario et au Québec et se sont poursuivies dans d'autres provinces au cours des dernières années. À la fin de 2010, le pourcentage des échantillons de viande de poulet contaminés par la bactérie Campylobacter variait de $21 \%$ au Québec à $42 \%$ en Colombie-Britannique (12).

Entre 2005 et 2010, le PICRA a observé une augmentation de la prévalence de la résistance à la ciprofloxacine parmi les bactéries Campylobacter provenant de la viande de poulet vendue au détail. Des différences importantes liées à la prévalence de la résistance dans les provinces ont été répertoriées; on a observé notamment une augmentation de la résistance en Colombie-Britannique (de $4 \%$ en 2007 à $17 \%$ en 2010) et en Saskatchewan (de $6 \%$ en 2005 à $11 \%$ en 2010). Elle est néanmoins demeurée relativement faible et stable dans les isolats provenant de viande de poulet vendue au détail recueillis en Ontario (de $4 \%$ en 2003 à $5 \%$ en 2010), au Québec (de $3 \%$ en 2003 à $2 \%$ en 2010) et dans les Maritimes (de $0 \%$ en 2008 à $4 \%$ en 2010) (12).

Plusieurs stratégies de communication ont été utilisées pour aviser les intervenants de la santé publique et vétérinaire de ces résultats. Au cours de l'été 2010, les ministères de la Santé et de l'Agriculture de la Colombie-Britannique et de la Saskatchewan en ont été avisés, et des vétérinaires spécialisés dans le domaine de la volaille ont été consultés. Au cours de l'été 2011, cette question fut abordée dans un bulletin de surveillance (13) du PICRA qui visait à informer l'industrie canadienne de la volaille, d'autres organismes du secteur de production primaire et des organismes vétérinaires, ainsi que des intervenants en santé publique. Une réunion a été organisée au cours de l'été 2013 à laquelle ont participé des intervenants de l'industrie de la volaille afin de leur fournir une mise à jour sur ces résultats. Lors de la réunion du PICRA avec les intervenants plurisectoriels nationaux en automne 2013, cette question a été examinée plus en profondeur, et des données récentes ont été présentées.

Le PICRA continue de surveiller la situation dans le cadre de ses activités de surveillance dans les fermes, en abattoir et dans le secteur de la vente au détail et observe toujours des changements dans les niveaux de résistance à la ciprofloxacine dans l'ensemble du pays. En 2012, et ce, depuis les premières notifications, la Colombie-Britannique et la Saskatchewan ont indiqué une diminution des niveaux de résistance à la ciprofloxacine parmi les isolats de Campylobacter provenant de viande de poulet vendue au détail, avec des prévalences de $8 \%$ et $5 \%$, respectivement. En 2012, cependant, une augmentation de la résistance a été signalée dans des régions où une résistance avait rarement été détectée auparavant. En Ontario, par exemple, la résistance a augmenté pour passer de $6 \%$ en 2011 à $16 \%$ en 2012, alors qu'au Québec, la résistance est passée de $0 \%$ en 2011 à $3 \%$ en 2012 (14). Cette observation a été confirmée par les données de surveillance en abattoir du PICRA, indiquant qu'une tendance similaire à la résistance à la ciprofloxacine a été observée parmi les isolats de Campylobacter provenant de poulets.

\section{Évaluation et mesures de santé publique}

La présence de Campylobacter résistant à la ciprofloxacine dans les poulets vendus au détail au Canada s'est accrue au cours des dernières années (2011 et 2012) (14), bien que l'ampleur et les tendances varient en fonction de la région. Les facteurs clés de l'émergence de Campylobacter résistant à la ciprofloxacine dans les échantillons de viande de poulet vendue au détail restent inconnus pour l'instant, mais l'utilisation de fluoroquinolones en dérogation des directives de l'étiquette dans le cas des poulets à griller, des poules reproductrices de type à griller, ainsi que des œufs d'incubation et poussins importés, est considérée comme un facteur contributif (12). 
D'autres facteurs pourraient être en cause. La documentation laisse entendre que des sources provenant des fermes mêmes ainsi que des facteurs opérationnels pourraient contribuer à la propagation de Campylobacter résistant aux antimicrobiens. Un examen systématique de la documentation semble indiquer que Campylobacter résistant aux antimicrobiens pourrait infiltrer un troupeau par les conduites d'eau potable puis se répandre dans toute la ferme par les ouvriers agricoles (par le port de chaussures contaminées) (15). Campylobacter aurait été propagé par des équipes itinérantes chargées de la collecte des poulets; ces équipes se déplacent de ferme en ferme, puis aux abattoirs, où les oiseaux sont exposés à de l'équipement contaminé lors du transport (15). L'abattage de poulets provenant de fermes où l'on a décelé la présence de la bactérie Campylobacter a été lié à la contamination des produits de viande de poulet $(11,13,14)$.

\section{Incidences sur la santé humaine}

Les infections à Campylobacter chez l'humain ont été associées à la consommation de produits de viande de volaille (16-19). Une étude menée aux États-Unis a montré qu'une infection causée par la bactérie Campylobacter résistante à la ciprofloxacine peut entraîner des infections prolongées (19). Plus récemment, une étude canadienne a révélé que les isolats de Campylobacter prélevés chez l'humain sont souvent génétiquement liés aux isolats provenant de la viande de poulet (20), mais les données de sensibilité pour les infections humaines sont limitées et ne sont pas accessibles aux systèmes de surveillance au Canada à l'heure actuelle.

Un ancien rapport d'évaluation des risques aux États-Unis (2000) a indiqué que les produits du poulet contaminés par Campylobacter résistant à la ciprofloxacine contribuent aux infections à Campylobacter résistant à la ciprofloxacine chez les humains (21). Par conséquent, les bonnes pratiques d'hygiène et de salubrité des aliments sont plus importantes que jamais puisqu'elles permettront de réduire la transmission de Campylobacter résistant et sensible, de la viande contaminée aux personnes. Cela comprend l'emballage et l'entreposage adéquats du poulet dans des réfrigérateurs, le nettoyage exhaustif des surfaces et des ustensiles qui sont entrés en contact avec du poulet cru, l'utilisation de différentes surfaces pour la préparation de la viande crue et des légumes et des fruits, ainsi qu'une cuisson complète (22).

\section{Incidence sur la politique de l'industrie}

Récemment, l'industrie canadienne de la volaille a élaboré une politique visant à éliminer l'utilisation préventive des antimicrobiens considérés comme étant de très haute importance en médecine humaine. Ils comprennent les céphalosporines de troisième génération et les antimicrobiens à base de fluoroquinolones (23). Cette politique est entrée en vigueur en mai 2014 pour les poulets à griller et le sera en mai 2015 pour les poules reproductrices de type à griller et les dindes (23). Une prolongation d'un an pour la mise en application de cette politique permettra à l'industrie d'informer les principaux intervenants de ce changement.

\section{Incidence sur la surveillance}

En avril 2013, le PICRA a introduit la surveillance de l'utilisation des antimicrobiens et de la résistance à ceux-ci à l'échelle des fermes de poulets à griller. Ce nouveau volet de surveillance appuiera l'étude axée sur les liens entre l'utilisation des antimicrobiens et la résistance à ceux-ci. II permettra le suivi de l'incidence potentielle des modifications récentes apportées à la politique de l'industrie en ce qui concerne la résistance de la bactérie Campylobacter et d'autres pathogènes entériques de la volaille.

\section{Discussion et conclusion}

Le PICRA a documenté que la bactérie Campylobacter résistante à la ciprofloxacine dans le poulet vendu au détail est présente partout au pays; que les niveaux dans l'ouest du Canada ont chuté, mais que les niveaux déjà faibles - ou inexistants - dans l'est du Canada ne cessent d'augmenter. La gestion du risque quant à la résistance aux antimicrobiens ne sera pas chose facile : les habitudes concernant l'utilisation des antimicrobiens peuvent changer très lentement, de même que la disparition des profils de résistance. Malgré les progrès réalisés, le PICRA continue de détecter la présence de 
Campylobacter résistant à la ciprofloxacine provenant du poulet vendu au détail au Canada. Nous ne pouvons pas encore établir comment la résistance aux fluoroquinolones est apparue dans les isolats de Campylobacter provenant de poulets à griller au Canada, ni la façon dont elle s'est propagée des régions de l'Ouest vers les régions de l'Est, en passant par les régions du Centre. II n'est également pas clair si cette résistance s'est développée de façon indépendante.

Le facteur clé de l'utilisation des fluoroquinolones dans cette industrie reste toujours difficile à déterminer. La Direction des médicaments vétérinaires a mis en place une politique sur l'utilisation des médicaments antimicrobiens de catégorie I en dérogation des directives de l'étiquette chez les animaux destinés à l'alimentation (10); toutefois, le respect de ces exigences relève de l'exercice de la médecine vétérinaire. Les données initiales provenant du programme lié à la ferme laissent entendre qu'une utilisation limitée des fluoroquinolones en dérogation des directives de l'étiquette a lieu chez les troupeaux de poulets à griller. Le PICRA continuera à surveiller l'usage des médicaments dans les fermes, ainsi que les raisons pour leur utilisation (c.-à-d. aux fins de prévention ou de traitement) et à évaluer les liens entre l'utilisation des antimicrobiens et la résistance à ceux-ci.

Le PICRA avait relevé des lacunes dans la surveillance de la bactérie Campylobacter et dans l'utilisation des antimicrobiens au Canada. Par conséquent, la surveillance de l'utilisation des antimicrobiens et la résistance à ceux-ci à l'échelle des fermes de poulets à griller au Canada a été introduite en avril 2013. Ces nouvelles données sont utilisées pour étudier les liens entre l'utilisation des antimicrobiens et la résistance à ceux-ci, de même que l'incidence des récents changements stratégiques. De plus, le PICRA détermine en ce moment la meilleure approche à adopter pour effectuer l'analyse de la résistance aux antimicrobiens causés par les infections à Campylobacter chez les humains, afin de mieux comprendre les répercussions sur la santé publique de l'exposition à Campylobacter résistant à la ciprofloxacine à partir de la viande de poulet vendue au détail et d'autres sources.

Le PICRA continuera à surveiller les tendances en matière d'utilisation d'antimicrobiens et la résistance à ceux-ci, à la suite de l'intervention par l'industrie visant à éliminer l'utilisation préventive des antimicrobiens de catégorie I (de très haute importance en médecine humaine). Toutefois, en suivant de bonnes pratiques d'hygiène et de préparation de la nourriture, les Canadiens peuvent réduire les risques de contracter une infection à Campylobacter provenant de la viande de poulet vendue au détail.

\section{Remerciements}

Les auteurs tiennent à remercier Rita Finley, la $D^{r e}$ Danielle Daignault ainsi que le personnel du Laboratoire de lutte contre les zoonoses d'origine alimentaire à Saint-Hyacinthe (Québec), Nicol Janecko et le personnel itinérant responsable de la vente au détail du PICRA; $D^{r}$ J.T. McClure/collaborateurs à l'Université de l'Île-du-Prince-Édouard et le Centre for Coastal Health en Colombie-Britannique. Les auteurs tiennent également à remercier l'Agence canadienne d'inspection des aliments et la Direction des médicaments vétérinaires de Santé Canada pour la révision du manuscrit.

\section{Financement}

Aucun financement externe n'a été reçu.

\section{Conflit d'intérêts}

II n'y a aucun conflit d'intérêts à déclarer.

\section{Références}

(1) Thomas, M.K. et Murray, R. au nom du groupe de travail chargé de l'estimation du fardeau des maladies d'origine alimentaire au Canada. Estimation du fardeau des maladies d'origine alimentaire au Canada. RMTC. 
2014 40; 14 :302-306. http://www.phac-aspc.gc.ca/publicat/ccdr-rmtc/14vol40/dr-rm40-14/dr-rm40-14-commfra.php

(2) Thomas MK, Murray R, Flockhart L, Pintar K, Pollari F, Fazil A, et al. Estimates of the burden of foodborne illness in Canada for 30 specified pathogens and unspecified agents, circa 2006. Foodborne Pathog Dis 2013; 10(7):639-48.

(3) Olson C, Ethelberg S, Van Pelt W, Tauxe R. Epidemiology of Campylobacter infections in industrialized nations. In: Nachamkin I, Szymanski CM, Blaser MJ, editors. Campylobacter. 3rd ed. Washington: American Society for Microbiology Press; 2008.

(4) Blondel-Hill E, Fryters S. Bugs and Drugs: An Antimicrobial/Infectious Diseases Reference. Edmonton: Alberta Health Services; 2012.

(5) Deckert AE, Reid-Smith RJ, Tamblyn SE, Morrell L, Seliske P, Jamieson FB, Irwin R, Dewey CE, Boerlin P, McEwen SA. Antimicrobial resistance and antimicrobial use associated with laboratory-confirmed cases of Campylobacter in two health units in Ontario. Can J Infect Dis Med Microbiol. 2013 Spring; 24(1):e16-e21.

(6) Dryden MS, Gabb RJ, Wright SK. Empirical treatment of severe acute community-acquired gastroenteritis with ciprofloxacin. Clin Infect Dis. 1996; 22:1019-25. DOI: 10.1093/clinids/22.6.1019.

(7) Organisation des Nations Unies pour l'alimentation et l'agriculture, Organisaton mondiale de la Santé, Organisation mondiale de la santé animale. Réunion mixte d'experts FAO/OMS/OIE sur les agents antimicrobiens d'importance critique. Rapport de la réunion d'experts FAO/OMS/OIE, FAO, Rome (Italie), 26-30 novembre 2007. http://www.fao.org/docrep/013/i0204f/i0204f00.pdf

(8) Gouvernement du Canada [page Web]. Santé Canada, Direction des médicaments vétérinaires. Catégorisation des médicaments antimicrobiens basée sur leur importance en médecine humaine. Avril 2009. http://www.hcsc.gc.ca/dhp-mps/vet/antimicrob/amr_ram_hum-med-rev-fra.php

(9) Hofacre CL, Fricke JA, Inglis T. Antimicrobial drug use in poultry. In: Giguere S, Prescott JF, Dowling PM, editors. Antimicrobial Therapy in Veterinary Medicine. 5th ed. John Wiley and Sons. Ames, lowa; 2013. p. 56987.

(10) Gouvernement du Canada [page Web]. Santé Canada, Direction des médicaments vétérinaires. Politique sur l'utilisation des médicaments en dérogation des directives de l'étiquette (UMDDE) chez les animaux destinés à l'alimentation. Août 2008. http://www.hc-sc.gc.ca/dhp-mps/vet/label-etiquet/pol_eldu-umdde-fra.php

(11) Agence de la santé publique du Canada (ASPC). Programme intégré canadien de surveillance de la résistance aux antimicrobiens (PICRA) : Rapport sommaire sur la résistance aux antimicrobiens 2011. Guelph (Ont.) : ASPC; 2012. http://publications.gc.ca/collections/collection_2013/aspc-phac/HP2-4-2-2011-fra.pdf

(12) Agunos A, Léger D, Avery BP, Parmley EJ, Deckert A, Carson CA, Dutil L. Ciprofloxacin resistant Campylobacter spp. in retail chicken, western Canada. Emerg Infect Dis. 2013 Jul; 19(7):1121-4. doi: 10.3201/eid1907.111417.

(13) Gouvernement du Canada [page Web]. Agence de la santé publique du Canada, Programme intégré canadien de surveillance de la résistance aux antimicrobiens (PICRA). Bulletin de surveillance : Augmentation de Campylobacter résistant à la ciprofloxacine dans la viande de poulet vendue au détail en Colombie-Britannique et en Saskatchewan. Novembre 2011. http://www.phac-aspc.gc.ca/cipars-picra/bulletin-fra.php

(14) Avery BP, Parmley EJ, Reid-Smith RJ, Daignault D, Finley, RL, Irwin, RJ. Canadian Integrated Program for Antimicrobial Resistance Surveillance: Retail food highlights, 2003-2012. CCDR. 2014 Nov 6; 40S-2:32-40. http://www.phac-aspc.gc.ca/publicat/ccdr-rmtc/14vol40/dr-rm40-S2/index-fra.

(15) Agunos A, Waddell L, Leger D, Taboada E. A systematic review characterizing on-farm sources of Campylobacter spp. for broiler chickens. PLoS One. 2014 Aug 29; 9(8):e104905.

(16) Roux F, Sproston E, Rotariu O, Macrae M, Sheppard SK, Bessell P, et al. Elucidating the aetiology of human Campylobacter coli infections. PLoS One. 2013 May 29; 8(5):e64504.

(17) Strachan NJ, Gormley FJ, Rotariu O, Ogden ID, Miller G, Dunn GM, et al. Attribution of Campylobacter infections in northeast Scotland to specific sources by use of multilocus sequence typing. J Infect Dis. 2009 Apr 15; 199(8):1205-8.

(18) Friedman CR, Hoekstra RM, Samuel M, Marcus R, Bender J, Shiferaw B, et al. Risk factors for sporadic Campylobacter infection in the United States: A case-control study in FoodNet sites. Clin Infect Dis. 2004 Apr 15; 38 Suppl 3:S285-96.

(19) Nelson JM, Smith KE, Vugia DJ, Rabatsky-Ehr T, Segler SD, Kassenborg HD, et al. Prolonged diarrhea due to ciprofloxacin resistant Campylobacter infection. J Infect Dis. 2004 Sep 15; 190(6):1150-7.

(20) Levesque S, Frost E, Arbeit RD, Michaud S. Multilocus sequence typing of Campylobacter jejuni isolates from humans, chickens, raw milk, and environmental water in Québec, Canada. JClin Microbiol. 2008 Oct; 46(10):3404-11. 
(21) United States Food and Drug Administration, Center for Veterinary Medicine. The human health impact of fluoroquinolone resistant Campylobacter attributed to the consumption of chicken. Washington: U.S. Food and Drug Administration, Center for Veterinary Medicine; 2000 Oct

18. http://www.fda.gov/downloads/AnimalVeterinary/SafetyHealth/AntimicrobialResistance/UCM083649.pdf [mis à jour le 5 janvier 2001].

(22) Gouvernement du Canada [page Web]. Campylobacter jejuni. Janvier 2012. http://canadiensensante.gc.ca/eating-nutrition/poisoning-intoxication/campylobacterfra.php? ga =1.187194152.1897391804.1401279223

(23) AgMedia Inc [page Web]. Better Farming. Canada's chicken farmers plan to eliminate some antibiotic use by May 2014. 2013 Dec 10. http://www.betterfarming.com/online-news/canada\%E2\%80\%99s-chicken-farmers-planeliminate-some-antibiotic-use-may-2014-54120 